\title{
Femoral Head Fovea Capitis Variant Configurations and Age Related Changes- A Radiological Study
}

\author{
Nihat Acar, ${ }^{1, *}$ Ahmet Karaarslan, ${ }^{2}$ Ahmet Karakasli, ${ }^{3}$ and Mehmet Erduran ${ }^{3}$ \\ ${ }^{1}$ Department of Orthopaedics and Traumatology, Catalca Ilyas Cokay Hospital, Catalca, Istanbul, Turkey \\ ${ }^{2}$ Department of Orthopedics and Traumatology, Sifa Hospital, 35260, Konak, Izmir, Turkey \\ ${ }^{3}$ Department of Orthopaedics and Traumatology, Dokuz Eylul University, Faculty of Medicine, 35340, Balcova, Izmir, Turkey \\ "Corresponding author: Nihat Acar, Department of Orthopaedics and Traumatology, Catalca Ilyas Cokay Hospital, Postalcode 34540, Catalca, Istanbul, Turkey. Tel: \\ +90-5375552636, E-mail: zenanacar@gmail.com
}

Received 2016 July 29; Revised 2016 November 15; Accepted 2016 December 26.

\begin{abstract}
Background: Many studies in the literature focused on the proximal geometry of the femur using cadaveric samples, X-rays or computed tomography, demonstrated substantial differences in the proximal femoral geometry in different patient groups. Many radiological parameters have been introduced in the literature to aid in diagnosis and surgical interventions.

Objectives: We aimed to assess the diameter of the femoral head fovea capitis in both genders with different age groups and to record their variant configurations.

Patients and Methods: Six-hundred true pelvis anteroposterior radiographs of patients were divided into three different age groups.

Results: In group 1, the general average values of fovea capitis index (FCI) in both female and male genders were $25.35 \% \pm 4.12 \%$ and $26.82 \pm 4.81 \%$, respectively $(\mathrm{P}=0.08)$. The general average $\mathrm{FCI}$ value of both sides was $26.08 \% \pm 4.46 \%$. In group 2 , the general average values of FCI values in both female and male genders were $26.69 \% \pm 5.27 \%$ and $26.88 \% \pm 4.77 \%$, respectively $(P=0.43)$. The general average FCI value of both sides was $26.78 \% \pm 4.93 \%$. In group 3 , the general average values of FCI value in both female and male genders were $28.86 \% \pm 4.98 \%$ and $29.16 \% \pm 3.82 \%$, respectively $(\mathrm{P}=0.27)$. The general average value of both sides was $28.93 \% \pm$ $4.40 \%$.

Conclusion: Fovea capitis size increases with aging process. It has four different configurations. Further clinical studies should be conducted to correlate the different configurations with other femoral head pathologies and anatomical variations.
\end{abstract}

Keywords: Femoral head, Fovea capitis, Fovea capitis index

\section{Background}

Proximal femoral geometry has gained so much attention during the last two decades (1-3). Variable radiological measures have been introduced to help radiologists and orthopedic surgeons to diagnose diseases, evaluate surgeries, and plan for surgical interventions (2-4). By introduction of picture archiving and communication systems (PACS) which became widespread and the standard radiological viewing tool in many orthopedic clinics, radiologists and orthopedic surgeons became more able to measure angles and distances even in a more precise manner $(5,6)$.

Many studies in the literature focused on the proximal geometry of the femur using cadaveric samples, X-rays or computed tomography, demonstrated substantial differences in the proximal femoral geometry in different patient groups. Many radiological parameters have been introduced in the literature to aid in diagnosis and surgical interventions $(4,7,8)$.

Few studies focused on the anatomical location of the fovea capitis in the femoral heads, while other researchers studied the anatomical variations of the ligamentum teres and its artery which has its terminal branches piercing the fovea capitis (9-13). However, it is strange that searching the literature did not reveal any study focusing on the fovea capitis variant configurations and size changes related to the aging process.

\section{Objectives}

The main purpose of this radiological study was twofold, to assess the diameter of the femoral head fovea capitis in different age groups and genders to determine its size related changes with aging process and to record the different anatomical configurations of the fovea capitis.

\section{Patients and Methods}

After getting the approval of the local ethics committee with a protocol number of 2070-GOA and decision 
number 2015/12-34, 600 true pelvis anteroposterior radiographs were obtained for patients between ages 20 and 80 years. The patients were divided into three groups of two hundreds. Group 1 patients were 20 to 39 years old (male, $n$ $=100$; female, $n=100)$, group 2 patients were 40 - 59 years old (male, $\mathrm{n}=100$; female, $\mathrm{n}=100$ ) and group 3 patients were 60 - 80 years old (male, $n=100$; female, $n=100$ ).

The inclusion criteria in this study were age between 20 and 80 years, no previous hip surgery, no metabolic bone disease history, no history of hip dysplasia, and no hip associated pathologies. A total of 600 hip pelvis AP views with 1200 hip views were obtained for this study.

To verify the normality assumption for each group associated subgroups of data, the goodness of fit test was used. The subgroups were found normally distributed in each group and there was no statistical significance between the left and the right sided parameters of each subgroup of the three groups with $\mathrm{P}>0.05$ in all cases.

To compare the values of the parameters between males, females and general values the unpaired t-tests were used, where the significance was set at $\mathrm{P}<0.05$.

\subsection{Radiological Measurement}

Fovea capitis index was introduced to eliminate the minimal magnification differences between the obtained true AP pelvis views. Fovea capitis index (Acar's index) was determined by measuring the diameter of the fovea capitis on true AP hip view from the upper border to the lower border of fovea capitis multiplied by 100 and divided by the femoral head diameter at its widest points; fovea capitis in$\operatorname{dex}($ Acar's index $)=($ fovea capitis diameter $(F C D) \times 100) \div$ (femoral head diameter) (Figure 1).

To make the description of variant fovea capitis configuration easy, the same terminologies used to describe the femoral head pathologies (including coxa magna, and coxa plana) were used to describe fovea capitis variant sizes.

Since the majority (higher than 97\% of the measured indexes or 1164 out of 1200) of fovea capitis indexes ranged between $18 \%$ and $35 \%$, fovea capitis with fovea capitis indexes smaller than $18 \%$ was called fovea capitis brevis; whereas, fovea capitis with fovea capitis indexes larger than 35\% were called fovea capitis magna.

The depth of each fovea capitis was recorded. The depth was measured by completing the head circle and measuring the distance between the deepest point of fovea capitis and the apex of the curve of the head circle passing over fovea capitis (Figure 2). The majority of the depths of fovea capitis (95.75\% of the measured fovea capitis depths or 1149 fovea depths), were between $2-4 \mathrm{~mm}$. Fovea capitis with fovea capitis depths smaller than $2 \mathrm{~mm}$ were called fovea plana; whereas, fovea capitis with fovea capitis depths larger than $4 \mathrm{~mm}$ were called fovea profunda.

All pelvis views were obtained with the beam of the Xray directed toward the midline above the symphysis pubis with both lower extremities in $15^{\circ}$ of internal rotation. All radiographs were taken using the same standard radiographic positioning using the same radiographic machine (Philips medical systems, digital diagnost). All measurements were accomplished using the extreme XDS digital program (extreme $\mathrm{PACS}^{\odot}$ company).

\section{Results}

In group 1 ( 20 - 39 years of age), the average value of the measured fovea capitis index in the female subgroup in the right hip was $24.58 \% \pm 3.44 \%$; whereas, it was $26.12 \%$ $\pm 4.81 \%$ in the left hip. No statistical significance was detected between the fovea capitis index (FCI) average values in the right and left hips. However, in the male subgroup, the average value of the measured $\mathrm{FCI}$ in the right hip was $25.29 \% \pm 4.27 \%$ and $28.35 \% \pm 5.34 \%$ for the left hip. A statistical significance was detected in the FCI between the right and left hips with a P value of 0.028 (Table 1). The general FCI average value in the female population was $25.35 \% \pm$ $4.12 \%$; whereas, it was $26.82 \% \pm 4.81 \%$ in the male population. No statistical significance was detected between the two general average values of groups. The general average value of FCI of both sides in both genders was $26.08 \% \pm$ $4.46 \%$ (Table 1 ).

In group 2, (40 - 59 years of age), the average value of the measured fovea capitis index in the female subgroup in the right hip was $25.73 \% \pm 4.82 \%$; whereas, it was $27.66 \%$ $\pm 5.37 \%$ in the left hip. No statistical significance was detected between the FCI average values in the right and left hips. However, in the male subgroup, the average FCI value in the right hip was $25.08 \% \pm 3.92 \%$ and $28.35 \% \pm 5.63 \%$ for the left hip. A statistical significance was detected in the FCI average values between the right and left hips with a $\mathrm{P}$ value of 0.02 . The general average FCI value in the female population was $26.69 \% \pm 5.27 \%$, while it was $26.88 \% \pm 4.77 \%$ in the male population. No statistical significance was detected between the two groups. The general average FCI value of both sides in both genders was $26.78 \% \pm 4.93 \%$.

In group 3 ( 60 - 80 years of age), the average measured fovea capitis index value in the female subgroup in the right hip was $27.66 \% \pm 4.67 \%$, while it was $29.71 \% \pm 5.29 \%$ in the left hip. No statistical significance was detected between the FCI average values in the right and left hips. However, in the male subgroup, the average FCI value in the right hip was $27.72 \% \pm 4.16 \%$ and $30.65 \% \pm 3.49 \%$ for the left hip. A statistical significance was detected in the FCI average values between the right and left hips with a P value 

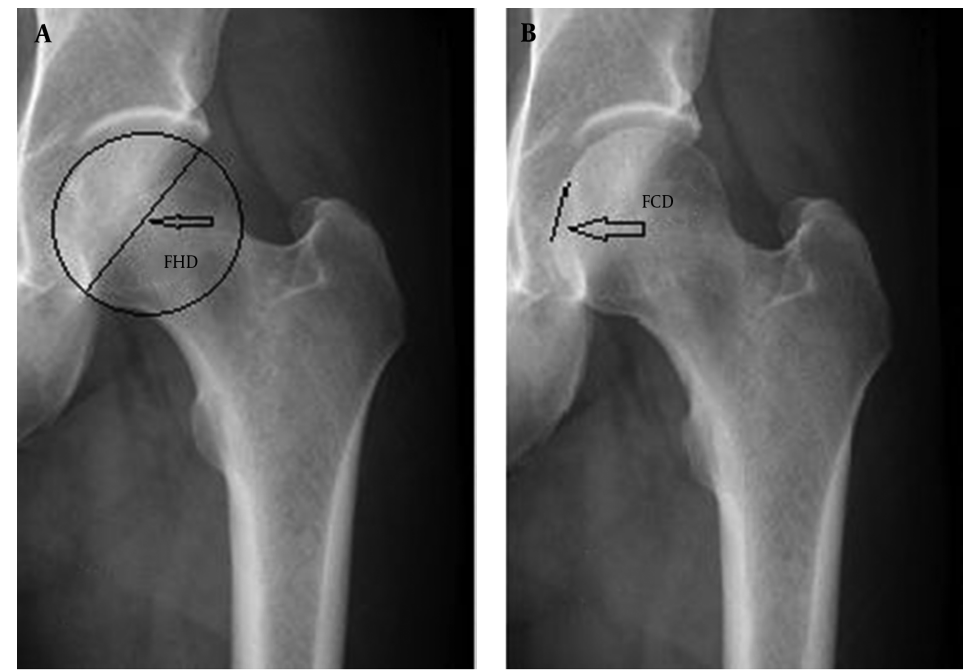

Figure 1. Anteroposterior view of the left hip joint A, This image shows the diameter of the femoral head by measuring the diameter of a complete circle around the femoral head. B, This image shows the diameter of fovea capitis by measuring the fovea capitis length from its upper margin to the lower margin. The fovea capitis index equation $(\mathrm{FCI})=($ fovea capitis diameter $(\mathrm{FCD}) \times 100) \div($ femoral head diameter $)$.
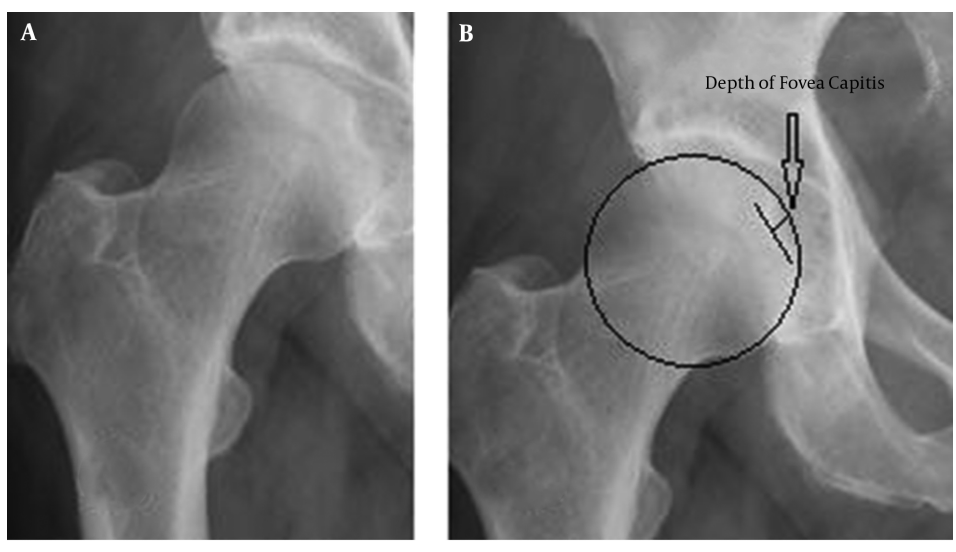

Figure 2. A, Anteroposterior view of the left hip joint. B, Measurement of the depth of the fovea capitis was done by completing the head circle and measuring the distance between the deepest point of fovea capitis and the apex of the curve of the head circle passing over the fovea capitis.

of 0.041. The general FCI average value in the female population was $28.68 \% \pm 4.98 \%$, while it is $29.16 \% \pm 3.82 \%$ in the male population. No statistical significance was detected between the two groups. The general average value of both sides in both genders was $28.93 \% \pm 4.40 \%$.

Analyzing the 1200 hip joints revealed a different four configuration of fovea capitis. During this study, the values of $97 \%$ of the FCI values (1164 out of 1200 hip values) were between $18 \%$ and $35 \%$. Whereas, the majority of fovea capitis depths (95.75\% of the measured fovea depths or 1149 fovea depths) ranged between $2 \mathrm{~mm}$ and $4 \mathrm{~mm}$.

The small sized fovea capitis, with a FCI less than $18 \%$, were called fovea brevis. They were discovered in six hips, composing $0.5 \%$ of all foveas (Figure 3 ). Whereas, $2.5 \%$ of the involved hips, 30 hips, had FCI values higher than 35\%, and were called fovea magna (Figure 4).

The shallow form of fovea capitis, where the depth is less than $2 \mathrm{~mm}$, is called fovea plana. These were discovered in 38 patients, composing 3.2\% of foveas (Figure 5).

The notched deepened form of fovea capitis, where the depth is more than $4 \mathrm{~mm}$, is called fovea profunda, discovered in 13 hips, composing 2.1\% of foveas (Figure 6). 
Table 1. Demonstration of the Femoral Head Fovea Capitis Index in Different Age Groups and Genders

\begin{tabular}{|c|c|c|c|c|c|c|c|}
\hline \multirow[t]{2}{*}{ Parameters } & \multicolumn{2}{|c|}{ Female } & \multicolumn{2}{|c|}{ Male } & \multirow{2}{*}{$\begin{array}{c}\text { Female General } \\
\text { Value }\end{array}$} & \multirow[b]{2}{*}{ Male General Value } & \multirow[b]{2}{*}{ General Value } \\
\hline & right & left & right & left & & & \\
\hline \multicolumn{8}{|c|}{ FCI \%, age (20 - 39), y } \\
\hline Mean \pm SD & $\begin{array}{c}24.58 \pm \\
3.44\end{array}$ & $\begin{array}{c}26.12 \pm \\
4.18\end{array}$ & $\begin{array}{c}25.29 \pm \\
4.27\end{array}$ & $\begin{array}{c}28.35 \pm \\
5.34\end{array}$ & $25.35 \pm 4.12$ & $26.82 \pm 4.81$ & $26.08 \pm 4.46$ \\
\hline Min & 17.3 & 20.7 & 17.8 & 20.1 & 17.3 & 20.1 & 17.3 \\
\hline Max & 28.5 & 31.2 & 32.6 & 34.3 & 31.2 & 34.3 & 34.3 \\
\hline PValue & \multicolumn{2}{|c|}{0.69} & \multicolumn{2}{|c|}{0.028} & \multicolumn{2}{|c|}{0.08} & \\
\hline \multicolumn{8}{|c|}{ FCI \%, age (40 - 59 ), y } \\
\hline Mean \pm SD & $\begin{array}{c}25.73 \pm \\
4.82\end{array}$ & $\begin{array}{c}27.66 \pm \\
5.37\end{array}$ & $\begin{array}{c}25.08 \pm \\
3.92\end{array}$ & $\begin{array}{c}28.69 \pm \\
5.63\end{array}$ & $26.69 \pm 5.27$ & $26.88 \pm 4.77$ & $26.78 \pm 4.93$ \\
\hline Min & 20.4 & 22.5 & 18.3 & 23.6 & 20.4 & 18.3 & 20.4 \\
\hline $\operatorname{Max}$ & 32.1 & 35.9 & 33.8 & 36.5 & 35.9 & 36.5 & 35.1 \\
\hline PValue & \multicolumn{2}{|c|}{0.17} & \multicolumn{2}{|c|}{0.02} & \multicolumn{2}{|c|}{0.43} & \\
\hline \multicolumn{8}{|c|}{ FCI \%, age (60 - 80$), y$} \\
\hline Mean \pm SD & $\begin{array}{c}27.66 \pm \\
4.67\end{array}$ & $\begin{array}{c}29.71 \pm \\
5.29 \\
\end{array}$ & $\begin{array}{c}27.72 \pm \\
4.16\end{array}$ & $\begin{array}{c}30.65 \pm \\
3.49\end{array}$ & $28.68 \pm 4.98$ & $29.16 \pm 3.82$ & $28.93 \pm 4.40$ \\
\hline Min & 21.4 & 25.2 & 22.8 & 23.6 & 21.4 & 22.8 & 22.5 \\
\hline Max & 34.7 & 38.6 & 36.2 & 36.3 & 38.6 & 36.3 & 37.4 \\
\hline P Value & \multicolumn{2}{|c|}{0.25} & \multicolumn{2}{|c|}{0.041} & \multicolumn{2}{|c|}{0.27} & \\
\hline
\end{tabular}

Abbreviation: FCI, fovea capitis index
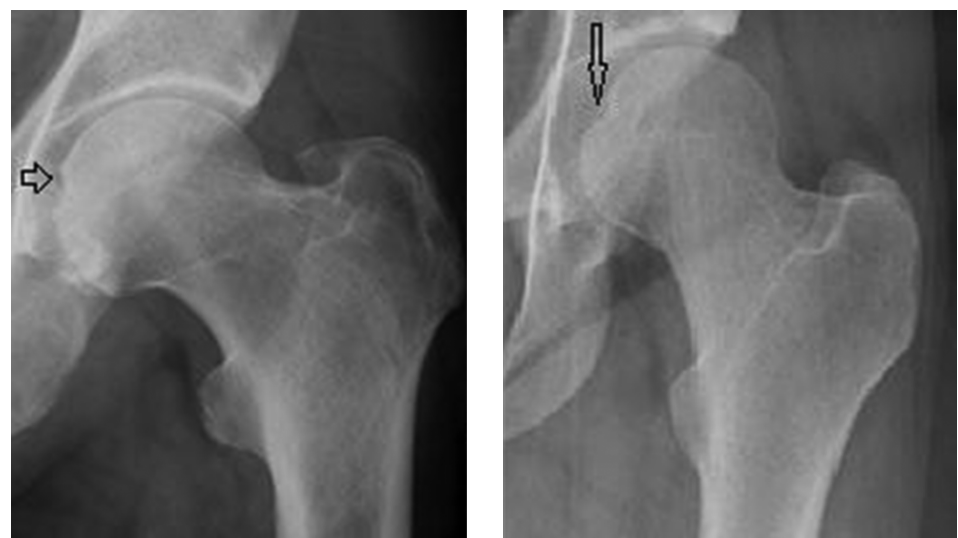

Figure 3. Black arrows show extra-small fovea capitis (Fovea Brevis) $(\mathrm{FCI}<18 \%)$

\section{Discussion}

The head of the femur is covered by articular cartilage, except for a small ovoid depression situated slightly inferior and posterior to the center of the head called fovea capitis, it serves as a site of attachment of ligamentum teres $(9,10)$.

Some studies have focused on the high location of fovea capitis. Nötzli et al. (11) has suggested that the abnormal superior position of the fovea capitis (fovea alta) is a radiological marker of hip dysplasia. He has demonstrated that fovea alta, which can be assessed by measurement of the delta angle, may be a predisposing factor for early acetabular perifoveal articular cartilage damage and may lead to an early osteoarthritis by diminishing the contact area of the femoral head with the superior weight- 

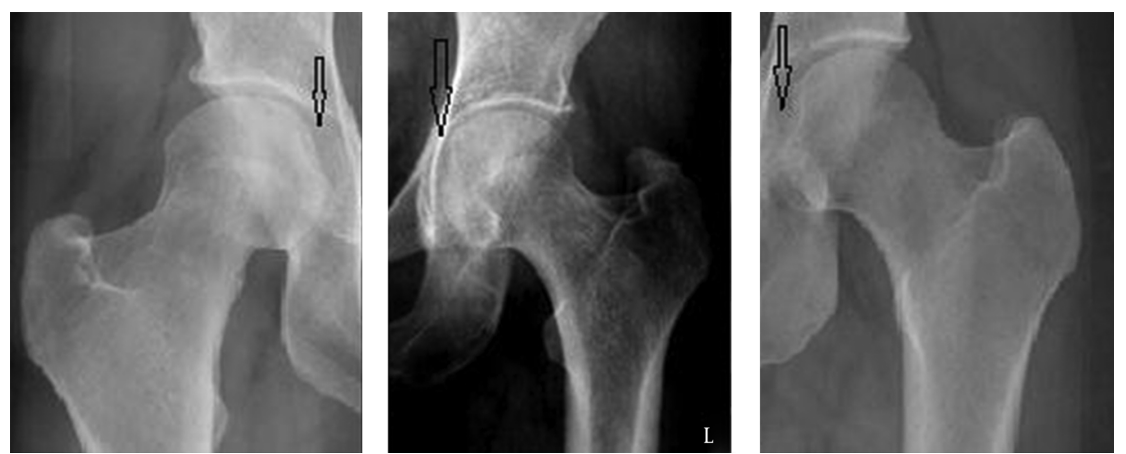

Figure 4. Black arrows show extra-large fovea capitis (fovea magna) (FCI > 35\%).
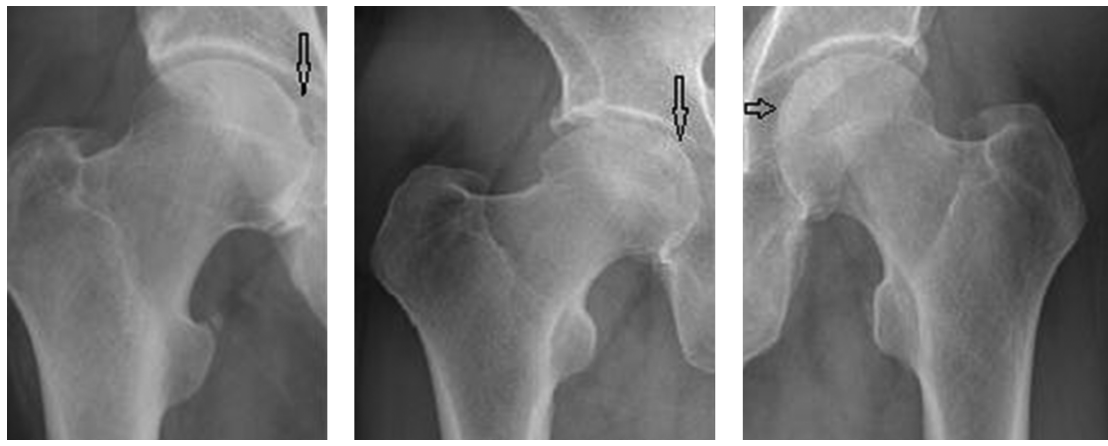

Figure 5. Black arrows show shallow fovea capitis (fovea plana).
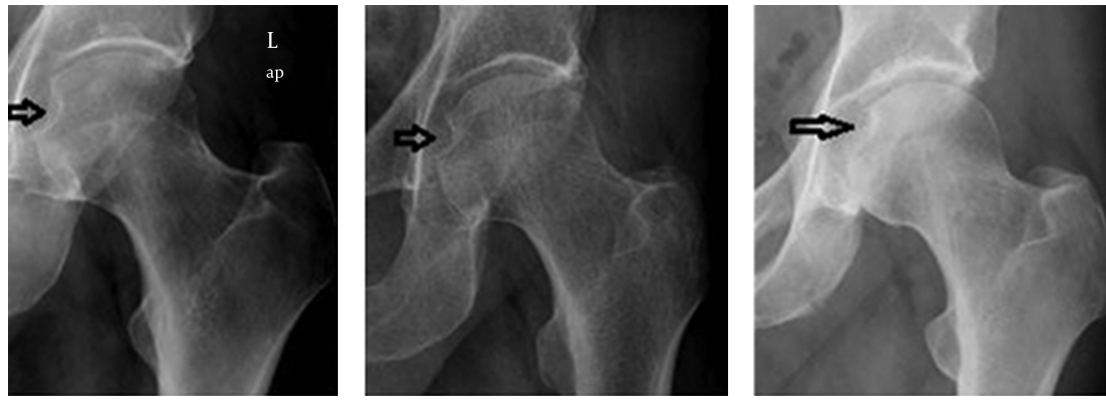

Figure 6. Black arrows show the notched form of fovea capitis (fovea profunda).

bearing articular surface of the acetabulum.

Another MRI study conducted by Beltran et al. (14) investigated the association between high fovea capitis (fovea alta) and hip dysplasia in young adults. They found a significant association between fovea alta and hip dysplasia.

Tucker et al. (15) conducted an apprehensive anatomical study on forty-four femora obtained from fresh cadavers by injecting the vessels piercing the fovea capitis by barium sulphate. They examined all specimens by obtaining a femoral head X-ray after decalcification.

They concluded that the foveolar vessels that arise either from the obturator or medial femoral circumflex arteries, or both, are present in every ligament examined, but the size varied considerably. In children, they recognized that the vessels were small and varied from one to five in number. Sometimes they form a network in the fovea capitis similar to the fingers of an outstretched hand. However, in adults, he noticed that the size alteration was strikingly larger than that of the pediatric vessels by $80 \%$. The foveo- 
lar vessels penetrated the osseous head through the fovea capitis, forming different anastomosis in more than $70 \%$ of specimens.

Fovea capitis contains the ligamentum teres (LT). Tan et al. (12) in his anatomical study concluded that LT has variable lengths and sometimes consists of multiple bundles. They also found that it is absent in $10 \%$ of individuals.

The exact function of LT is still not well understood. However, in a recent study performed by Martin et al. (13), they reported that LT is composed of a variant bundle number and it has a stabilizing role in internal and external rotations and tractions of the hip joint.

This study was conducted to assess the diameter changes of fovea capitis between both genders in different age groups. The design of the measurement, which was used in this study, is stemmed from our extensive clinical experience in hip surgery. It is strange not to find any anatomical or radiological well-designed studies in the literature focusing on the fovea capitis diameter changes in both genders with aging process.

The fovea capitis index $(\mathrm{FCI})$ equation $=$ (fovea capitis diameter $($ FCD $) \times 100) \div($ femoral head diameter $)$ is valid, since it is suggested to avoid errors related to minimal magnification variations between pelvis views.

Although there was no statistical significance between the $1^{\text {st }}$ and $2^{\text {nd }}$ groups, the $2^{\text {nd }}$ group FCI average value was larger than that of the $1^{\text {st }}$ group. The $3^{\text {rd }}$ group demonstrated the highest FCI average value when compared to the $1^{\text {st }}$ and $2^{\text {nd }}$ group's average values with a significant statistical difference, $(\mathrm{P}=0.016$, and 0.032 respectively).

Statistical analysis of fovea capitis index of the femoral head revealed that the fovea capitis diameter increases with aging process. The process of fovea capitis enlargement with aging process can be explained by degeneration of the perifoveal thin cartilage rim around fovea capitis.

The average values of FCI of the left hip were obviously larger than the average values of the FCI of the right hip, with a statistical significant difference between the resulted values. This can be explained mostly by the smaller left femoral head diameter compared to the right ones as demonstrated before by many researchers (16-18).

Many pathologies may affect the femoral head which is the crucial part of the hip joint. The femoral head contains very few radiological landmarks, one of which is the fovea capitis. The clinical significance of such anatomical variation of fovea capitis configurations has not been researched before. However, some inquiries have been produced by this study worthy to be investigated.

Further clinical and radiological studies should be conducted to correlate the association of fovea capitis different configurations with some well-known anatomical features and pathologies of the femoral head including ab- sent, short or hypertrophied ligamentum teres, ischemic or trauma-related avascular necrosis (AVN) and absent or active ligamentum teres blood supply.

However, we acknowledge the presence of some limitations in this study. X-ray views were used while computerized tomography (CT) can be more sensitive and accurate for measurements. However, $\mathrm{CT}$ is more expensive and exposes patients to more radiation than traditional X-ray. Despite the fact that this radiological study does not carry meaningful clinical results, it opens the field for investigations to further assess these anatomical variations and to reveal their impact on clinical practice.

In conclusion, fovea capitis size increases with aging process, it has four configurations, the clinical significance of such variant figures should be investigated clinically to detect their association with femoral head pathologies.

\section{Footnotes}

Authors' Contributions: Dr. Nihat Acar, concept of the study, application and measurment and writting of the manuscript; Dr. Ahmet Karaarslan, statistics of the study; Dr. Ahmet Karaksli, measurments and writing; Dr. Mehmet Erduran, literature review and structure of the study.

Conflict of interest: All authors declare that there is no conflict of interest regarding this study.

Financial Disclosure: None declared.

Funding/Support: None declared.

\section{References}

1. Kay RM, Jaki KA, Skaggs DL. The effect of femoral rotation on the projected femoral neck-shaft angle. J Pediatr Orthop. 2000;20(6):736-9. doi: 10.1097/01241398-200011000-00007. [PubMed: 11097245].

2. Beaule PE, Allen DJ, Clohisy JC, Schoenecker P, Leunig M. The young adult with hip impingement: deciding on the optimal intervention. J Bone Joint Surg Am. 2009;91(1):210-21. [PubMed: 19122097].

3. Steppacher SD, Tannast M, Ganz R, Siebenrock KA. Mean 20-year followup of Bernese periacetabular osteotomy. Clin Orthop Relat Res. 2008;466(7):1633-44. doi: 10.1007/s11999-008-0242-3. [PubMed: 18449617].

4. Acar N, Harb A, Albaya A, Kaskin H. The clinical results of a novel method for minimal invasive dynamic hip screw fixation of intertrochanteric fractures compared to the conventional one. Eur $J$ Trauma Emerg Surg. 2016 doi: 10.1007/s00068-016-0690-5. [PubMed: 27277071].

5. Johnson LJ, Cope MR, Shahrokhi S, Tamblyn P. Measuring tip-apex distance using a picture archiving and communication system (PACS). Injury. 2008;39(7):786-90. doi: 10.1016/j.injury.2007.12.019. [PubMed: 18539280].

6. Gnudi S, Ripamonti C, Lisi L, Fini M, Giardino R, Giavaresi G. Proximal femur geometry to detect and distinguish femoral neck fractures from trochanteric fractures in postmenopausal women. Osteoporos Int. 2002;13(1):69-73. doi: 10.1007/s198-002-8340-2. [PubMed: 11878458]. 
7. Crabtree N, Lunt M, Holt G, Kroger H, Burger H, Grazio S, et al. Hip geometry, bone mineral distribution, and bone strength in European men and women: the EPOS study. Bone. 2000;27(1):151-9. doi: 10.1016/S8756-3282(00)00300-8. [PubMed: 10865223].

8. Greendale GA, Young JT, Huang MH, Bucur A, Wang Y, Seeman T. Hip axis length in mid-life Japanese and Caucasian U.S. residents: no evidence for an ethnic difference. Osteoporos Int. 2003;14(4):320-5. doi: 10.1007/s00198-002-1367-y. [PubMed: 12730747].

9. Philippon MJ, Rasmussen MT, Turnbull TL, Trindade CA, Hamming MG, Ellman MB, et al. Structural Properties of the Native Ligamentum Teres. Orthop J Sports Med. 2014;2(12):2325967114561962. doi: 10.1177/2325967114561962. [PubMed: 26535290].

10. Perumal V, Woodley SJ, Nicholson HD. Ligament of the head of femur: A comprehensive review of its anatomy, embryology, and potential function. Clin Anat. 2016;29(2):247-55. doi: 10.1002/ca.22660. [PubMed: 26480296].

11. Notzli HP, Muller SM, Ganz R. [The relationship between fovea capitis femoris and weight bearing area in the normal and dysplastic hip in adults: a radiologic study]. Z Orthop Ihre Grenzgeb. 2001;139(6):502-6. doi: 10.1055/s-2001-19231. [PubMed: 11753770].

12. Tan CK, Wong WC. Absence of the ligament of head of femur in the hu- man hip joint. Singapore Med J. 1990;31(4):360-3. [PubMed: 2124003].

13. Martin HD, Hatem MA, Kivlan BR, Martin RL. Function of the ligamentum teres in limiting hip rotation: a cadaveric study. Arthroscopy. 2014;30(9):1085-91. doi: 10.1016/j.arthro.2014.04.087. [PubMed: 24908256].

14. Beltran LS, Mayo JD, Rosenberg ZS, De Tuesta MD, Martin O, Neto LS, et al. Fovea alta on MR images: is it a marker of hip dysplasia in young adults?. AJR Am J Roentgenol. 2012;199(4):879-83. doi: 10.2214/AJR.11.8193. [PubMed: 22997382].

15. Tucker FR. Arterial supply to the femoral head and its clinical importance. J Bone Joint Surg Br. 1949;31B(1):82-93. [PubMed:18124373].

16. Rawal B, Ribeiro R, Malhotra R, Bhatnagar N. Anthropometric measurements to design best-fit femoral stem for the Indian population. Indian J Orthop. 2012;46(1):46-53. doi: 10.4103/0019-5413.91634. [PubMed: 22345806].

17. Siwach RC, Dahiya S. Anthropometric study of proximal femur geometry and its clinical application. Indian J Orthop. 2003;37(4):247.

18. Atilla B, Oznur A, Caglar O, Tokgozoglu M, Alpaslan M. [Osteometry of the femora in Turkish individuals: a morphometric study in 114 cadaveric femora as an anatomic basis of femoral component design]. Acta Orthop Traumatol Turc. 2007;41(1):64-8. [PubMed: 17483639]. 